\title{
More Than Just Heartburn: Does Famotidine Effectively Treat Patients with COVID-19?
}

\author{
Robert W. Malone ${ }^{1}$ \\ Accepted: 20 January 2021 / Published online: 24 February 2021 \\ (c) The Author(s), under exclusive licence to Springer Science+Business Media, LLC part of Springer Nature 2021
}

Famotidine is an exquisitely specific inverse agonist of the human histamine $\mathrm{H} 2$ receptor. This agent is primarily administered as a treatment for gastroesophageal reflux disease (GERD) and related foregut disorders attributable to acid hypersecretion. Early in the COVID-19 outbreak, multiple research groups employing computational docking algorithms (computer programs that predict the interactions between small molecules and proteins at the atomic level) identified famotidine as a potential competitive inhibitor of both principal proteases expressed by the novel human coronavirus SARS-CoV-2. These groups posited that this remarkably safe, inexpensive, off-patent (generic) drug might directly inhibit replication of SARS-CoV-2, providing clinical benefit for COVID-19 patients. Subsequent non-clinical bench research has clearly demonstrated that famotidine does not bind to either protease to a significant extent and does not inhibit SARS-CoV-2 replication (see [1] for summary). Many of the unique clinical symptoms observed during the early phase of COVID-19 are consistent with known effects of histamine release. Most likely, if famotidine reduces the severity for COVID-19, it acts via its antagonism or inverse agonism of histamine signaling and arrestin-biased activation [1]. Multiple retrospective studies, case series, or reports have since been performed seeking to clarify whether famotidine treatment improves clinical outcomes in outpatient or in newly admitted inpatient cohorts suffering from COVID-19 disease. In some retrospective analyses, significant benefit has been reported, while others conclude no benefit.

In this issue of Digestive Diseases and Sciences, Drs. Sun, Chen, and colleagues report the first rigorous metaanalysis of data abstracted from peer-reviewed and published retrospective studies available up to the time of analysis (October 2020) [2]. Their findings clearly establish that

Robert W. Malone

rwmalonemd@gmail.com

1 RW Malone MD LLC, Madison, VA, USA famotidine administration at the standard GERD treatment doses (20-40 mg/day) does not provide a significant benefit in reducing the risk of serious illness, death, and intubation for hospitalized COVID-19 patients. Although not available to Sun, Chen et al. at the time of their analysis, an independent retrospective study by the Janssen/Johnson \& Johnson team of Drs. Shoaibi, Fortin et al. [3] has further confirmed and extended these findings.

Retrospective analyses of clinical datasets are one of the most powerful tools available to rapidly test whether drug repurposing "hits" have clinical merit. The retrospective analysis approach is straightforward, and the logic is compelling: If a repurposed candidate can be identified, and the pharmaceutical is prescribed at sufficient frequency (for another indication such as GERD) in patients suffering from a disease (such as COVID-19), then a retrospective analysis may be useful. As clinical patient data accumulate, sufficient cases exposed to both the repurposed drug candidate and the disease may support analysis of potential association. Nevertheless, this approach presumes that the dosing regimen (timing, route, and level) for the common indication (GERD) is within the therapeutic window required for the hypothesized new clinical indication (COVID-19). To illustrate, it is unrealistic and naïve to assume that the dosing regimen for an over-the-counter indication would be the same as for a life-threatening hyper-inflammatory response to a novel infectious pathogen since the dosing, pharmacokinetics, and pharmacodistribution recommended for the licensed indication may not align with that required for the repurposed clinical indication.

To avoid artifacts, retrospective propensity score analysis requires sufficient knowledge of confounding variables in order to support statistical correction and adjustment. For example, in the case of COVID-19 and patients with GERD, one might assume that patients receiving first-line therapy with proton pump inhibitors (PPI) and also suffering from COVID-19 might serve as a valid control population for patients receiving second-line GERD therapy (famotidine). 
Such assumptions may not withstand the test of time, as is the case with PPI $[4,5]$, which thereby introduced artifacts into at least one relevant retrospective analyses [6]. Therefore, the promise and potential value of retrospective analyses for evaluating repurposed drug candidates must be viewed with caution.

The story of how famotidine became a priority drug repurposing candidate for COVID-19 began with SARSCoV-2 infection of a physician/scientist leading one of the computational docking teams focused on the papain-like protease of SARS-CoV-2. This occurred during a Cambridge Mass drug discovery conference immediately after the Biogen super-spreader event (late February 2020). Having developed COVID-19 disease, the investigator began self-administering famotidine, which the team had identified as a therapeutic PLpro inhibitor candidate. Famotidine provided almost immediate improvements in the symptoms of shortness of breath and lung "burning." The investigator self-titrated to $60 \mathrm{mg}$ oral administration three times daily, discontinued treatment after seven days, experienced renewed symptoms and then re-initiated treatment for an additional seven days. The investigator and team had been working closely with personnel employed by both the US DoD and HHS on identifying repurposed drug candidates for COVID-19 and reported the findings to US government officials within both agencies. The office of the US Health and Human Services (HHS) Assistant Secretary for Preparedness and Response (BARDA) elected to fund a clinical inpatient trial of famotidine as a potential COVID-19 therapeutic. Original plans to perform an intravenous doseranging study in newly hospitalized patients were scrapped when the institutional review board insisted on including hydroxychloroquine in the treatment arms (NCT04370262). After enrollment was initiated, changes in standard of care, Good Clinical Practice (GCP) audit irregularities, and failure to enroll adequate numbers of patients compromised that study (now listed as completed); as a likely consequence, results have yet to be reported. Unfortunately, since other randomized clinical trials were postponed while awaiting the results of this HHS-funded study, a prospective randomized dose-ranging single agent study may never be performed.

A modest case series report from an affiliated outpatient study (NCT04389567) concluded that optimal symptomatic relief from COVID-19 required famotidine be administered at $60 \mathrm{mg}$ PO three times a day, as had been initially observed. Higher dosage levels are also supported by detailed examination of the mechanism of action, pharmacology and pharmacokinetics of famotidine as a potential COVID-19 therapeutic [1].

At this time, neither adequately powered prospective randomized clinical trials nor retrospective studies have examined whether famotidine administered at $60 \mathrm{mg}$ PO thrice daily or above provide clinical benefit, but this dosage level or above has nevertheless gained informal acceptance worldwide due to anecdotal positive clinical responses and practicing physician referrals.

Drs. Sun, Chen, and colleagues have established that the standard GERD famotidine dose of $20-40 \mathrm{mg}$ PO per day does not provide clinical benefit for COVID-19 disease in hospitalized patients. Unresolved is whether higher famotidine dosage levels predicted to mitigate histamine H2-receptor-mediated effects during SARS-CoV-2 infection can provide clinical benefit in similar patient populations. Unfortunately, since virtually all leading research groups employing famotidine in treatment protocols have migrated to multi-agent strategies [7-9], the baseline efficacy and effectiveness of high-dose famotidine as a single agent for treatment of COVID-19 may never be known.

\section{Reference}

1. Malone RW, Tisdall P, Fremont-Smith P, et al. COVID-19: famotidine, histamine, mast cells, and mechanisms. Frontiers in Pharmacology. 2021 (in Press).

2. Sun S, Chen Y, Hu L, Wu Y, Liang, M, Ahmed MA, Bhan D, Guo A, Yang H, Zuo Y, Yan Y, Zhou Q. Does Famotidine reduce the risk of progression to severe disease, death, and intubation for COVID-19 patients? A systemic review and meta-analysis. Digestive Diseases and Sciences. (Epub ahead of print). doi: https ://doi.org/10.1007/s10620-021-6872-2.

3. Shoaibi A, Fortin S, Weinstein R, Berlin JA, Ryan P. Comparative effectiveness of famotidine in hospitalized COVID-19 patients. American Journal of Gastroenterology. 2022 (in Press).

4. Lee SW, Ha EK, Yeniova A, Oet al. Severe clinical outcomes of COVID-19 associated with proton pump inhibitors: a nationwide cohort study with propensity score matching Gut. 2021;70:76-84.

5. Almario CV, Chey WD, Spiegel BMR. Increased Risk of COVID19 Among Users of Proton Pump Inhibitors Am J Gastroenterol. 2020;115:1707-1715.

6. Freedberg DE, Conigliaro J, Wang TC et al. Famotidine use is associated with improved clinical outcomes in hospitalized COVID-19 patients: a propensity score matched retrospective cohort study. Gastroenterology. 2020.

7. Kory P, Meduri GU, Iglesias J, Varon J, Marik PE. Clinical and scientific rationale for the "MATH+" hospital treatment protocol for COVID-19. J Intensive Care Med. 2021;36:135-156

8. Hogan RB, Hogan RB, Cannon T, et al. Dual-histamine receptor blockade with cetirizine-famotidine reduces pulmonary symptoms in COVID-19 patients. Pulmonary Pharmacology \& Therapeutics. 2020:101942.

9. Tomera KM, Malone R, Kittah JK. Hospitalized COVID-19 patients treated with celecoxib and high dose famotidine adjuvant therapy show significant clinical responses Frontiers in Pharmacology. 2021 (in Press).

Publisher's Note Springer Nature remains neutral with regard to jurisdictional claims in published maps and institutional affiliations. 